\title{
Analysis of the Spike Proteins Suggest Pangolin as an Intermediate Host of COVID-19 (SARS-CoV-2)
}

\author{
Sohail Raza ${ }^{\dagger 1}$, Muhammad Tariq Navid ${ }^{\dagger 2}$, Wajeeha Zahir ${ }^{3}$, Muhammad Nabeel Khan ${ }^{4}$, Muhammad Awais ${ }^{5}$, Tahir \\ Yaqub $^{1}$, Masood Rabbani ${ }^{1}$, Muhammad Rashid ${ }^{6}$, Salina Saddick ${ }^{7}$ and Muhammad Asif Rasheed ${ }^{* 3}$ \\ ${ }^{1}$ Institute of Microbiology, University of Veterinary and Animal Sciences, Lahore 54000, Pakistan \\ ${ }^{2}$ Department of Biological Sciences, National University of Medical sciences, Rawalpindi 46000, Pakistan \\ ${ }^{3}$ Department of Biosciences, COMSATS University Islamabad, Sahiwal Campus, 57000, Sahiwal, Pakistan \\ ${ }^{4}$ DHQ Teaching Hospital, Sahiwal Medical College, 57000, Sahiwal, Pakistan \\ ${ }^{5}$ University Institute of Biochemistry and Biotechnology, Pir Meher Ali Shah Arid Agriculture University, Rawalpindi, Pakistan \\ ${ }^{6}$ Institute of Virology, University of Karachi, Pakistan \\ ${ }^{7}$ Department of Biological Sciences, Faculty of Science, King Abdulaziz University, Jeddah 21589, Saudi Arabia \\ *For correspondence: asif.rasheed@cuisahiwal.edu.pk \\ †Contributed equally to this work and are co-first authors
}

Received 05 November 2020; Accepted 30 November 2020; Published 25 January 2021

\begin{abstract}
The novel coronavirus (SARS-CoV-2) is a third member of its group that has introduced public health catastrophes around the globe. Since, its emergence in Wuhan, China by December 2019, SARS-CoV-2 infected millions of human population along with many casualties globally. The transmission potential of SARS-CoV-2 between humans has already been studied. Despite this transmission in human population, the primary origin of SARS-CoV-2 has been linked with bats by the help of an intermediate (secondary) host. This study was assumed to investigate the possible secondary or intermediate host to shuttle down the SARS-CoV-2 transmission and further to mitigate future pandemics. The antigenic surface/spike (S) protein was used for the structural and genomic analysis through currently available computer assisted technology. For the In-silico analysis, 43 sequences of S-protein of coronaviruses originated in various species were retrieved from nucleotide database of NCBI. These sequences were matched to find any similarities/differences by employing pairwise and multiple sequence alignment. The phylogenetic analysis was conducted to observe the relation among different species through MEGA software. Finally, comparative analysis for structures of S-protein (superimposition) was done with reference structure by using UCSF Chimera software. The results of this study expressed maximum match of S-protein sequences of human coronavirus with Bat and with Pangolin sequences respectively. The Phylogenetic analysis between Bat and Pangolin showed that SARS-CoV-2 transmitted from bats to humans possibly through the intermediate host of Pangolin. (C) 2021 Friends Science Publishers
\end{abstract}

Keywords: Bat; Human; Intermediate host; Pangolin; SARS-CoV-2

\section{Introduction}

The current novel coronavirus disease (COVID-19) emerged in Wuhan, China and captured the globe within four months, thus declaring global health emergency. During the last couple of decades, the coronaviruses have emerged with sever outbreaks i.e., Severe Acute Respiratory Syndrome coronaviruses (SARS-CoV) in 2003 and Middle East Respiratory Syndrome coronaviruses (MERS-CoV) in 2012. The COVID-19 is a third type of coronavirus that is inducing high morbidity and mortality in humans.

Since its initial outbreak in Wuhan, China, COVID-19 has infected more than 2.0 million of individuals with more than 0.12 million deaths and spread over 205 countries so far. The causative organism of COVID-19 is a betacororonavirus that is named as SARS-CoV-2. It is a zoonotic virus that transmits from animals to humans. Generally, the coronaviruses are enveloped viruses having a spherical or pleomorphic shape and contain positive-sense RNA. The genome is ranged between 26 to $32 \mathrm{Kbps}$ with $80-120 \mathrm{~nm}$ of diameter (Fung and Liu 2019). The viral genome contains four structural proteins i.e., Envelop protein (E), Spike protein (S), Membrane protein (M) and Nucleo-capsid protein (N) (Schoeman and Fielding 2019). Each of the proteins has an important role in virus life cycle as $\mathrm{S}$-protein is for attachment to host cell, N-protein is responsible for nucleocapsid formation, and $\mathrm{M} \&$ E-proteins have roles in viral assembly (Masters 2006; McBride et al. 2014; Kirchdoerfer et al. 2016). 
One of the important challenges is to determine the origin of SARS-CoV-2, to understand its transmission from animals to humans. Now it has been established that SARSCoV-2 has been originated from horseshoe bats (Zhou et al. 2020), but based on the transmission of earlier Coronavirus the bats cannot able to transfer the virus directly to the humans. For example, SARS-CoV, is the closest relative of SARS-CoV-2 that caused Severe Acute Respiratory Syndrome (SARS) pandemic in 2003. It was also transmitted from bats through intermediate host "the masked palm civet" and finally to humans. Likewise, MERS, the Coronavirus that caused Middle East Respiratory Syndrome caused by Coronavirus in 2012, transmitted from bats to humans through intermediate host dromedary camel (Reusken et al. 2016). The human-animal interface is playing a major role in cross-species viral transmission and providing an appropriate place for various gene recombinations; thus introducing new variants. The COVID-19 is supposed to be the product of the recombination of various coronaviruses that existed for a long time in various hosts and generate its ability to adapt to human hosts. However, in this long journey, what kind of intermediate hosts have been used by coronaviruses to reach humans? It is entirely important to find the answer to broaden our understandings of the emergence and potential transmission of COVID-19. The identification of the intermediate host is a mystery for the spread of COVID- 19. By knowing the fact of viral spread, the researchers may be able to prevent further spread of the pandemic.

In this study, to identify the intermediate host, the genetic and structural analyses of Coronaviruses (including SARS-CoV-2) spike proteins were performed using bioinformatic approaches. The results of genetic and structural analysis are predicted the pangolin as a proximate intermediate host of SARS-CoV-2.

\section{Materials and Methods}

\section{Retrieval of glycoprotein sequences}

The sequences of the spike protein of coronaviruses were retrieved from the National Center for Biotechnology Information (NCBI) nucleotide database. These sequences belong to different species including humans. A total of 43 sequences from different species were retrieved. The list of the species and the accession numbers are mentioned in Table 1.

\section{Pairwise alignment}

The retrieved sequences were aligned to check the similarities and differences of the retrieved sequences against a reference sequence (Human Coronavirus Sequence). All the retrieved sequences were aligned against the reference coronavirus sequence using the BLAST algorithm.

\section{Multiple sequence alignment}

The multiple sequence alignment of all the retrieved sequences was performed to check the similarities and differences among the sequences. Moreover, multiple sequence alignment is required to perform phylogenetic analysis as well. The multiple sequence alignment of all the sequences was performed using Clustal Omega webserver (Sievers and Higgins 2014).

\section{Phylogenetics analysis}

To highlight the relationship among different species, phylogenetics analysis was performed by Molecular Evolutionary Genetics Analysis (MEGA) 6.06 software tool (Tamura et al. 2013). Moreover, to confirm the results, phylogenetics analysis was performed by different algorithms including parsimony analysis, maximum likelihood analysis and unweighted pair group method with arithmetic mean (UPGMA) analysis.

\section{Protein structure prediction and refinement}

The protein structures of all the retrieved sequences mentioned in Table 1 were predicted either by homology modeling or threading algorithms. First of all, the templates for the sequences were searched in Protein Databank (PDB) using the BLAST algorithm. The structures of all proteins were predicted by homology modeling where a good template was found in PDB. Moreover, the structures of the remaining proteins were predicted by threading where good templates of the proteins were not found. Furthermore; it was ensured that the quality of the structures is worthy. The qualities of the predicted protein structures were enhanced by Modrefiner web server for those proteins where quality was a little compromised (Xu and Zhang 2011).

\section{Proteins' structure superimposition}

To perform protein structure alignment, the superimpositions of the structures were performed by UCSF Chimera 1.14 software (Goddard et al. 2005). Just like pairwise alignment, the predicted structures were aligned to check the similarities and differences of the predicted structures against the reference structure. The human coronavirus glycoprotein structure was taken as reference and all the predicted structures were compared against it.

\section{Results}

\section{Pairwise Alignment}

The pairwise alignment of all the retrieved sequences was performed against the reference human coronavirus sequence. The results of the pairwise sequence alignment are mentioned in Table 2. According to the results, the 
Table 1: The sequences of spike protein retrieved from NCBI nucleotide database. Altogether, 43 sequences of different species were retrieved

\begin{tabular}{lll}
\hline Sr. No. & ACCESION NO & ORGANISM \\
\hline 1 & JF792617 & Rat coronavirus \\
2 & JF792616 & Rat coronavirus \\
3 & NC_012936 & Rat coronavirus \\
4 & FJ938068 & Rat coronavirus \\
5 & NC_032730 & Rat coronavirus \\
6 & KF294380 & Rat coronavirus \\
7 & KT368891 & Camel coronavirus \\
8 & JF792615 & Dromedary camel coronavirus \\
9 & JF792614 & Dromedary camel coronavirus \\
10 & NC_012937 & Dromedary camel coronavirus \\
11 & KT368892 & Bovine coronavirus \\
12 & JF792613 & Bovine coronavirus \\
13 & JF792612 & Bovine coronavirus \\
14 & MH043953 & Bovine coronavirus \\
15 & MH043952 & Bovine coronavirus \\
16 & AF220295 & Bovine coronavirus \\
17 & AF353511 & Porcine epidemic diarrhea virus \\
18 & KP890336 & Porcine epidemic diarrhea virus \\
19 & MG546690 & Porcine epidemic diarrhea virus \\
20 & MG546687 & Porcine epidemic diarrhea virus \\
21 & MF807952 & Porcine epidemic diarrhea virus \\
22 & MF807951 & Porcine epidemic diarrhea virus \\
23 & MF782687 & Porcine epidemic diarrhea virus \\
24 & KF663561 & Infectious bronchitis virus \\
25 & KF663560 & Infectious bronchitis virus \\
26 & KF663559 & Infectious bronchitis virus \\
27 & KC008600 & Infectious bronchitis virus \\
28 & KX272465 & Infectious bronchitis virus \\
29 & MK878536 & Infectious bronchitis virus \\
30 & KP981644 & Canine coronavirus \\
31 & GQ477367 & Canine coronavirus \\
32 & AY307021 & Canine coronavirus \\
33 & AY307020 & Canine coronavirus \\
34 & KY938558 & Bat coronavirus \\
35 & NC_009988 & Bat coronavirus \\
36 & EF203067 & Bat coronavirus \\
37 & EF203066 & Bat coronavirus \\
38 & EF203065 & Bat coronavirus \\
39 & EF203064 & Bat coronavirus \\
40 & KX442565 & Bat coronavirus \\
41 & KX442564 & Bat coronavirus \\
42 & QHD43416 & Human coronavirus \\
43 & (Zhang et al., 2020a) & Pangolin coronavirus \\
\hline & &
\end{tabular}

sequence similarity against the reference sequence was found maximum in Bat1 specie while the least similarity was found in Bvirus4. The results were arranged based on the alignment score. Hence, Bat1 secured an alignment score of 1843 with $97 \%$ query coverage, $72.76 \%$ identity and $0 \mathrm{E}$ values. These figures highlighted that the result were significant with minimum noise. Moreover, Pangolin got the second-highest similarity score to compare with reference human coronavirus sequence. Pangolin secured 1620 score for alignment with $88 \%$ query coverage, $88.62 \%$ identity, and $0 \mathrm{E}$ values. Furthermore, Bvirus 4 got the least similarity against the reference sequence. Bvirus4 secured 30.4 scores of alignment with just $1 \%$ query coverage, $83.33 \%$ identity and $5.4 \mathrm{E}$ values which highlights that the similarity of the sequence is very less compared to the reference sequence.
Table 2: The pairwise alignment results of all the retrieved sequences. The alignment was performed against the reference human coronavirus sequence. The maximum similarity was found in Bat1 specie while the least similarity was found in Bvirus4. The data was arranged on the basis of alignment score

\begin{tabular}{|c|c|c|c|c|c|}
\hline Sr. No. & Organism & Total Score & Query Cover & E value & Identity \\
\hline 1 & bat1 & 1843 & $97 \%$ & 0 & $72.76 \%$ \\
\hline 2 & pangolin & 1620 & $88 \%$ & 0 & $88.62 \%$ \\
\hline 3 & bovine 3 & 579 & $73 \%$ & $1.00 \mathrm{E}-151$ & $37.68 \%$ \\
\hline 4 & bovine4 & 578 & $73 \%$ & $2.00 \mathrm{E}-151$ & $37.68 \%$ \\
\hline 5 & bovine2 & 578 & $73 \%$ & $3.00 \mathrm{E}-151$ & $37.68 \%$ \\
\hline 6 & bovine 5 & 577 & $73 \%$ & $5.00 \mathrm{E}-151$ & $37.68 \%$ \\
\hline 7 & bat7 & 574 & $79 \%$ & 0 & $35.94 \%$ \\
\hline 8 & bovine1 & 573 & $73 \%$ & $1.00 \mathrm{E}-148$ & $37.55 \%$ \\
\hline 9 & bovine 6 & 569 & $73 \%$ & $2.00 \mathrm{E}-147$ & $37.15 \%$ \\
\hline 10 & camel4 & 568 & $75 \%$ & $2.00 \mathrm{E}-146$ & $36.63 \%$ \\
\hline 11 & camel3 & 568 & $91 \%$ & $2.00 \mathrm{E}-146$ & $36.50 \%$ \\
\hline 12 & camel2 & 568 & $91 \%$ & $2.00 \mathrm{E}-146$ & $36.50 \%$ \\
\hline 13 & camell & 567 & $91 \%$ & $3.00 \mathrm{E}-146$ & $36.50 \%$ \\
\hline 14 & bat8 & 561 & $79 \%$ & $5.00 \mathrm{E}-180$ & $35.03 \%$ \\
\hline 15 & rat5 & 545 & $71 \%$ & $1.00 \mathrm{E}-140$ & $36.69 \%$ \\
\hline 16 & rat2 & 545 & $71 \%$ & $1.00 \mathrm{E}-140$ & $36.69 \%$ \\
\hline 17 & rat4 & 541 & $71 \%$ & $3.00 \mathrm{E}-139$ & $36.30 \%$ \\
\hline 18 & rat3 & 541 & $71 \%$ & $3.00 \mathrm{E}-139$ & $36.30 \%$ \\
\hline 19 & rat1 & 541 & $71 \%$ & $3.00 \mathrm{E}-139$ & $36.30 \%$ \\
\hline 20 & rat6 & 381 & $79 \%$ & $3.00 \mathrm{E}-107$ & $29.42 \%$ \\
\hline 21 & Bvirus6 & 373 & $44 \%$ & $2.00 \mathrm{E}-102$ & $37.31 \%$ \\
\hline 22 & bat6 & 367 & $63 \%$ & $8.00 \mathrm{E}-101$ & $31.14 \%$ \\
\hline 23 & bat2 & 367 & $63 \%$ & $8.00 \mathrm{E}-101$ & $31.14 \%$ \\
\hline 24 & virus7 & 367 & $66 \%$ & $3.00 \mathrm{E}-92$ & $30.33 \%$ \\
\hline 25 & bat5 & 365 & $63 \%$ & $2.00 \mathrm{E}-100$ & $31.23 \%$ \\
\hline 26 & bat4 & 365 & $63 \%$ & $3.00 \mathrm{E}-100$ & $31.23 \%$ \\
\hline 27 & bat3 & 365 & $63 \%$ & $3.00 \mathrm{E}-100$ & $31.23 \%$ \\
\hline 28 & virus1 & 365 & $65 \%$ & $9.00 \mathrm{E}-91$ & $30.44 \%$ \\
\hline 29 & Bvirus5 & 357 & $39 \%$ & $1.00 \mathrm{E}-106$ & $38.36 \%$ \\
\hline 30 & virus5 & 349 & $60 \%$ & 7.00E-93 & $30.33 \%$ \\
\hline 31 & virus3 & 347 & $58 \%$ & 8.00E-93 & $30.33 \%$ \\
\hline 32 & virus4 & 347 & $60 \%$ & $2.00 \mathrm{E}-92$ & $30.16 \%$ \\
\hline 33 & canine 4 & 345 & $56 \%$ & $5.00 \mathrm{E}-101$ & $31.61 \%$ \\
\hline 34 & canine 3 & 342 & $56 \%$ & $7.00 \mathrm{E}-100$ & $30.80 \%$ \\
\hline 35 & canine1 & 340 & $56 \%$ & $3.00 \mathrm{E}-99$ & $32.07 \%$ \\
\hline 36 & virus6 & 340 & $60 \%$ & $3.00 \mathrm{E}-90$ & $29.63 \%$ \\
\hline 37 & canine 2 & 339 & $56 \%$ & 7.00E-99 & $31.82 \%$ \\
\hline 38 & virus2 & 337 & $60 \%$ & $1.00 \mathrm{E}-89$ & $30.35 \%$ \\
\hline 39 & Bvirus1 & 31.6 & $1 \%$ & 3.7 & $66.67 \%$ \\
\hline 40 & Bvirus2 & 31.6 & $1 \%$ & 3.6 & $66.67 \%$ \\
\hline 41 & Bvirus3 & 30.4 & $1 \%$ & 5.4 & $83.33 \%$ \\
\hline 42 & Bvirus4 & 30.4 & $1 \%$ & 5.4 & $83.33 \%$ \\
\hline
\end{tabular}

\section{Multiple sequence alignment}

The results of multiple sequence alignment are shown in Fig. 1. According to the results, the maximum length of the sequence was related to Canine3 (1481 amino acids) specie while the minimum length was related to Poultry 1 (224 amino acids) specie. Moreover, stars in the alignment highlight the conserved residues among all the compared species based on multiple sequence alignment. Although no conserved residues were found in multiple sequence alignment results when we compared all 43 sequences. Some amino acid residues were conserved when we limited the alignment to some species. For example, if we limit the species to Rat, Camel, and Bovine, then the sequences remained conserved at many points. This highlights the 


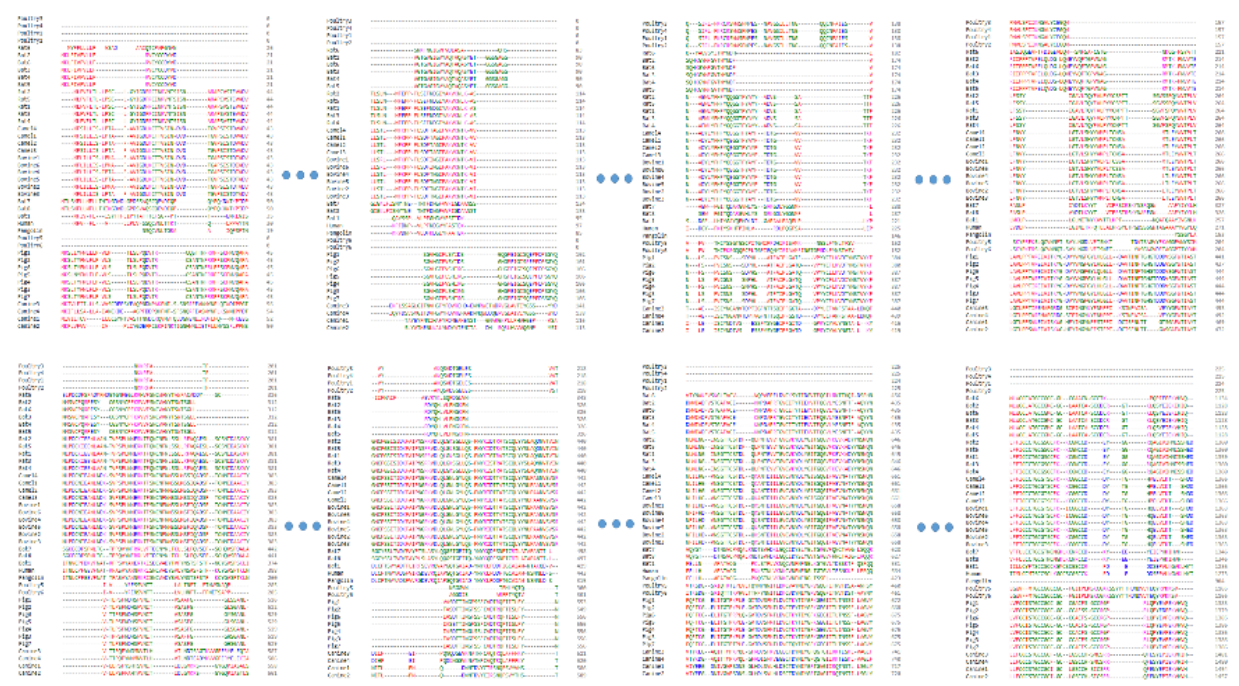

Fig. 1: The results of multiple sequence alignment. The maximum length of the sequence was found in Canine 3 with 1481 amino acids while specie while minimum length was found in Bvirus1 with 224 amino acids specie
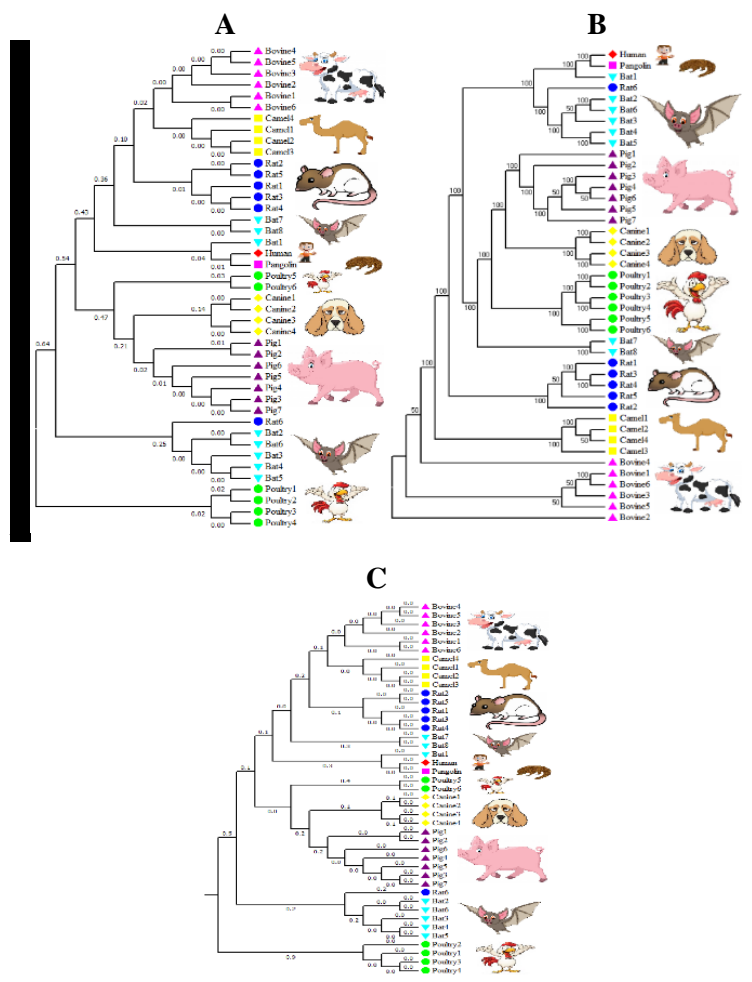

Fig. 2: The phylogenetics analysis of all species included in the current study. The results were confirmed by three different algorithms including Maximum Likelihood (A), Maximum Parsimony (B) and UPGMA (C). All results highlighted that human corona virus has maximum relation with pangolin. The analysis was performed by MEGA 6.06 software

similarity of coronavirus sequences among these three species. Hence, there is a possibility that Rat, Camel, and Bovine directly infected themselves during the transmission of coronavirus.
$\mathbf{A}$

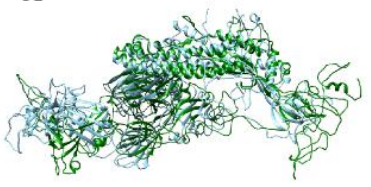

C

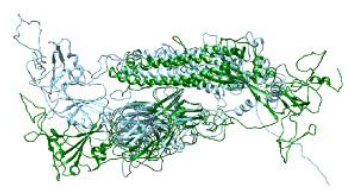

B

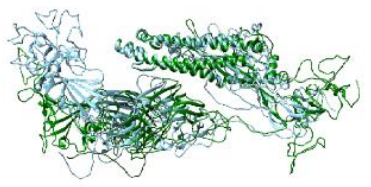

D

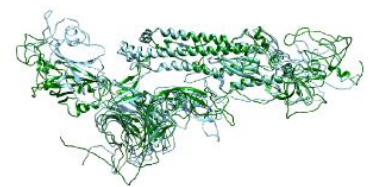

Fig. 3: Comparative structures analysis for spike protein structures of all the species in present study against human SARS-CoV-2 spike protein (structure shown in green). The predicted structures of all the proteins were compared against reference structure of human corona virus glycoprotein by superimposition. $\mathbf{A}=$ Bovine 1. $\mathbf{B}=$ Camel 1. $\mathbf{C}=$ Rat1. $\mathbf{D}=$ Bat1. Amongst these species, the maximum similarity among the structures was found in Bat1

\section{Phylogenetics analysis}

The phylogenetics analysis was performed by MEGA 6.06 software. To confirm the results, the analysis was performed by three different algorithms including Parsimony, Maximum Likelihood, and UPGMA. The clusters in tree were formed according to species. For example, bovines were present in one cluster; camels were present in another cluster. Moreover, rats, bats and canine were present in their respective cluster as shown in Fig. 2. Interestingly, all results clustered human, bat, and pangolin in one cluster. This cluster is of great significance as we are focusing on human coronavirus origin. Moreover, in this cluster, the human had a closer relationship with pangolin compared to Bat1. These results highlight the possibility that the origin of human 

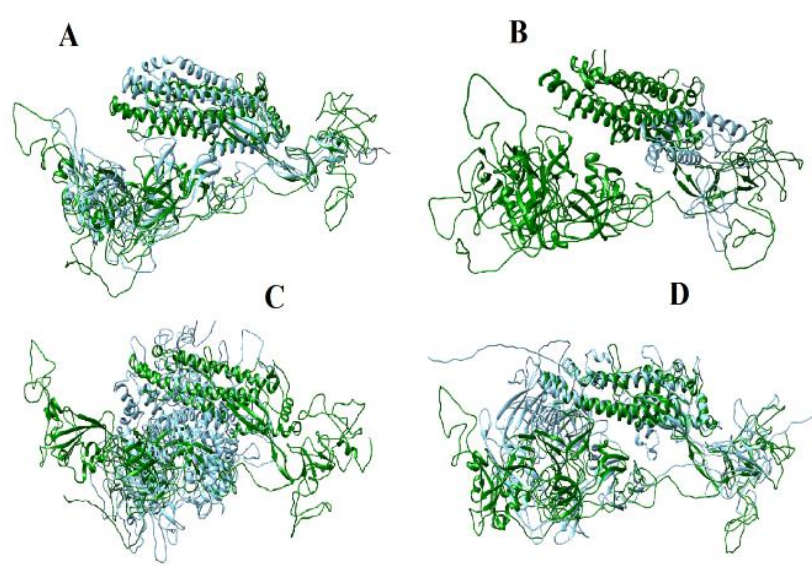

Fig. 4: Comparative structures analysis for spike protein structures of all the species in present study against human SARSCoV-2 spike protein structure (structure shown in green). The predicted structures of all the proteins were compared against reference structure of human corona virus glycoprotein by superimposition. $\mathbf{A}=$ Pangolin. $\mathbf{B}=$ Poultry 1. $\mathbf{C}=$ Canine $1 . \mathbf{D}=$ Pig 1. Amongst these species, the maximum similarity among the structures was found in Pangolin

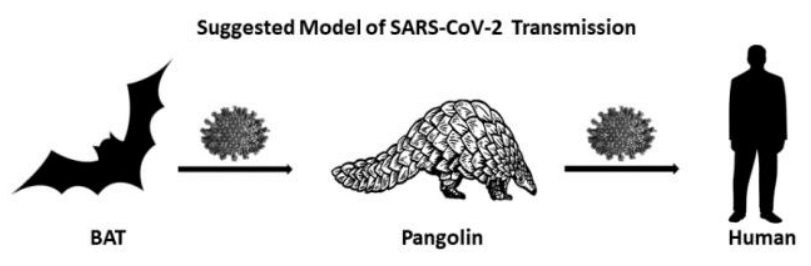

Fig. 5: The results of present study suggest the possible role of pangolin as an intermediate host of transferring SARS-CoV-2 from bats to humans

coronavirus is from Bat to Pangolin to Human (Fig. 5).

\section{Protein structures analysis}

The predicted structures of all the proteins were compared against the reference structure of human coronavirus glycoprotein. The structure comparison is shown in Fig. 3 and Fig. 4. According to the comparison, the maximum similarity among the structures was found in Pangolin species while Bats also got significant similarities against the Human glycoprotein structure. Moreover, if we compare the resemblance of structures among different species, then Pangolin and Bats got maximum similarities in the structure compared with humans than the rest of the species. This result strengthens the findings of pairwise alignment and phylogenetic analysis where Pangolin and Bats showed the highest similarities against humans.

\section{Discussion}

An earlier study claimed that snakes were likely to be the intermediate hosts of the SARS-CoV-2. Hence, at Wuhan
Huanan Seafood Market, the researchers compared the virus in eight animals. The codon usage in the SARS-CoV-2 virus was compared in these animals. The researchers found the similar codon usage pattern in snakes compare to SARSCoV-2. Hence, they declared snakes might likely intermediate hosts for the virus (Ji et al. 2020). However, in a follow-up study, three coronaviruses (SARS-CoV-2, SARS-CoV and MERS-CoV) were compared. The investigators found more than 10,000 different kinds of animals in their studies. Hence, they rejected the earlier claim of snake-borne transmission of SARS-CoV-2 (Zhang et al. 2020a).

The spike protein of SARS-CoV-2 is the most important concerning viral infection. The spike protein is the outermost protein of the virus that involves the attachment of the virus to the cell receptors called Angiotensin Converting Enzyme 2 (ACE2). The ACE2 is the transmembrane receptor on the mammalian cells that is utilized by SARS-CoV-2 for infection. Therefore, the spike protein involved in the infectivity and host specificity of the coronaviruses and the good target to find the possible origin of the SARS-CoV-2 (Letko et al. 2020). In this study, 42 sequences of Coronaviruses spike protein from different host species were compared with the SARS-CoV-2 spike protein to find the possible intermediate host. The bats are still the probable host of origin for SARS-CoV-2 (Zhang et al. 2020c). The multiple sequence alignment results of all spike protein against SARS-CoV-2 spike protein exhibited that pangolin Coronavirus spike protein has a maximum similarity of $88.62 \%$ with SARS-CoV-2 as compared to bat Coronavirus, which exhibited similarity of $72.76 \%$. This sequence similarity of SARS-CoV-2 with pangolin Coronavirus is very high as the spike protein is the main protein that binds the cell receptor thus determines the host specificity. Moreover, there are only 5 amino acid sequences that are different on Receptor Binding Domain (RBD) of pangolin spike protein as compared to bat coronavirus, which has 19 different amino acids on Receptor Binding Domain (RBD) of the spike protein. Recently, a published study shows similar results and predicts pangolin as the intermediate host (Zhang et al. 2020a). The phylogenetic analysis of spike protein using three different algorithms confirm the above findings that humans, bat and pangolin coronaviruses were found in the same cluster, however; spike protein of pangolin virus has a close relation with human Coronavirus than bat coronavirus. These results suggest the possible role of pangolin as an intermediate host of transferring SARS-CoV-2 from bats to humans as suggested in figure 5. Similar results were reported in a recently published study (Zhang et al. 2020b). To further examine our findings, we performed the structural analysis by comparing the spike protein structure of human Coronavirus with other coronaviruses. As previously predicted, the human Coronavirus spike protein is more closely related to the pangolin Coronavirus than bat or other any coronaviruse. Taking all together, the MSA, 
phylogenetic analysis and structural analysis predict pangolin as an intermediate host. As the virus originates from the live food market Wuhan, where wild animals including bats and the pangolin were kept together that provide the best environment of Coronavirus transfer between hosts.

\section{Conclusion}

Amid the COVID-19 outbreak, the detailed understanding of how the SARA-CoV-2 transfers to humans will be helpful in the prevention of future outbreaks. The SARSCoV-2 transfers from bats to humans through an intermediate host. Using the genetic and structural analysis of spike proteins from different coronaviruses, we predict that pangolins served as an intermediate host to transfer the novel virus from bats to humans.

\section{Author Contributions}

Sohail Raza and Muhammad Tariq Navid wrote the paper. Wajeeha Zahir, Muhammad Nabeel Khan and Muhammad Awais performed the analysis. Tahir Yaqub, Masood Rabbani, Muhammad Rashid and Salina Saddick reviewed the paper. Muhammad Asif Rasheed conceived the idea and reviewed the paper.

\section{References}

Fung TS, DX Liu (2019). Human coronavirus: Host-pathogen interaction. Annu Rev Microbiol 73:529-557

Goddard TD, CC Huang, TE Ferrin (2005). Software extensions to Ucsf chimera for interactive visualization of large molecular assemblies. Structure 13:473-482
Ji W, W Wang, X Zhao, J Zai, X Li (2020). Cross-species transmission of the newly identified coronavirus 2019-Ncov. J Med Virol 92:433-440

Kirchdoerfer RN, CA Cottrell, N Wang, J Pallesen, HM Yassine, HL Turner, KS Corbett, BS Graham, JS Mclellan, AB Ward (2016). Prefusion structure of a human coronavirus spike protein. Nature $531: 118-121$

Letko M, A Marzi, V Munster (2020). Functional assessment of cell entry and receptor usage for Sars-Cov-2 and other lineage B betacoronaviruses. Nat Microbiol 5:562-569

Masters PS (2006). The molecular biology of coronaviruses. Adv Virol Res 66:193-292

Mcbride R, M Van Zyl, BC Fielding (2014). The coronavirus nucleocapsid is a multifunctional protein. Viruses 6:2991-3018

Reusken CB, VS Raj, MP Koopmans, BL Haagmans (2016). Cross host transmission in the emergence of mers coronavirus. Curr Opin Virol 16:55-62

Schoeman D, BC Fielding (2019). Coronavirus envelope protein: Current knowledge. Virol $J$ 16:69-90

Sievers F, DG Higgins (2014). Clustal omega, accurate alignment of very large numbers of sequences. Meth Mol Biol 1079:105-116

Tamura K, G Stecher, D Peterson, A Filipski, S Kumar (2013). Mega6: Molecular evolutionary genetics analysis version 6.0. Mol Biol Evol 30:2725-2729

$\mathrm{Xu} \mathrm{D,} \mathrm{Y} \mathrm{Zhang} \mathrm{(2011).} \mathrm{Improving} \mathrm{the} \mathrm{physical} \mathrm{realism} \mathrm{and} \mathrm{structural}$ accuracy of protein models by a two-step atomic-level energy minimization. Biophys $J$ 101:2525-2534

Zhang C, W Zheng, X Huang, EW Bell, X Zhou, Y Zhang (2020a). Protein structure and sequence reanalysis of 2019-Ncov genome refutes snakes as its intermediate host and the unique similarity between its spike protein insertions and Hiv-1. J Proteom Res 19:1351-1360

Zhang J, W Jia, J Zhu, B Li, J Xing, M Liao, W Qi (2020b). Insights into the cross-species evolution of 2019 novel coronavirus. J Infect 80:671-693

Zhang T, Q Wu, Z Zhang (2020c). Probable Pangolin Origin Of Sars-Cov-2 Associated With The Covid-19 Outbreak. Curr Biol 30:1346-1351

Zhou P, XL Yang, XG Wang, B Hu, L Zhang, W Zhang, HR Si, Y Zhu, B Li, CL Huang, HD Chen, J Chen, Y Luo, H Guo, RD Jiang, MQ Liu, Y Chen, XR Shen, X Wang, XS Zheng, K Zhao, QJ Chen, F Deng, LL Liu, B Yan, FX Zhan, YY Wang, GF Xiao, ZL Shi (2020). A pneumonia outbreak associated with a new coronavirus of probable bat origin. Nature 579:270-273 